\title{
Modified LS Method for Unconstrained Optimization*
}

\author{
Jinkui Liu' ${ }^{1}$,i Zheng ${ }^{2}$ \\ ${ }^{1}$ College of Mathematics and Computer Science, Chongqing Three Gorges University, \\ Chongqing, China \\ ${ }^{2}$ Chongqing Energy College, Chongqing, China \\ E-mail: liujinkui2006@126.com \\ Received December 28, 2010; revised May 6, 2011; accepted May 9, 2011
}

\begin{abstract}
In this paper, a new conjugate gradient formula $\beta_{k}^{V S S}$ and its algorithm for solving unconstrained optimization problems are proposed. The given formula $\beta_{k}^{V L S}$ satisfies $\beta_{k}^{V L S} \geq 0$ with $d_{k}$ satisfying the descent condition. Under the Grippo-Lucidi line search, the global convergence property of the given method is discussed. The numerical results show that the new method is efficient for the given test problems.
\end{abstract}

Keywords: Unconstrained Optimization, Conjugate Gradient Method, Grippo-Lucidi Line Search, Global Convergence

\section{Introduction}

The primary objective of this paper is to study the global convergence properties and practical computational performance of a new conjugate gradient method for nonlinear optimization without restarts, and with suitable conditions. lem:

Consider the following unconstrained optimization prob-

$$
\min _{x \in R^{n}} f(x),
$$

where $f: R^{n} \rightarrow R$ is smooth and its gradient $g$ is available. LS conjugate gradient method for solving unconstrained optimization problem is iterative formulas of the form

$$
\begin{gathered}
x_{k+1}=x_{k}+\alpha_{k} d_{k}, \\
d_{k}= \begin{cases}-g_{k}, & \text { for } k=1 ; \\
-g_{k}+\beta_{k} d_{k-1}, & \text { for } k \geq 2,\end{cases}
\end{gathered}
$$

where $x_{k}$ is the current iterate, $\alpha_{k}$ is a positive scalar and called the steplength which is determined by some line search, $d_{k}$ is the search direction; $g_{k}$ is the gradient of $f$ at $x_{k}$, and $\beta_{k}$ is a scalar and

$$
\beta_{k}^{L S}=-\frac{g_{k}^{\mathrm{T}}\left(g_{k}-g_{k-1}\right)}{d_{k-1}^{\mathrm{T}} g_{k-1}}, \text { (Liu-Storey (LS) [1]), }
$$

[2] proved the global convergence of the LS method

"This work was supported by The Nature Science Foundation of Chongqing Education Committee (KJ091104, KJ101108). with Grippo-Lucidi line search. And the Grippo-Lucidi line search is to compute

$$
\alpha_{k}=\max \left\{\rho^{j} \frac{\tau\left|g_{k}^{\mathrm{T}} d_{k}\right|}{\left\|d_{k}\right\|^{2}}, j=0,1,2, \cdots\right\}
$$

satisfying :

$$
\begin{gathered}
f\left(x_{k}+\alpha_{k} d_{k}\right)-f\left(x_{k}\right) \leq-\delta \alpha_{k}^{2}\left\|d_{k}\right\|^{2}, \\
-c_{2}\left\|g_{k+1}\right\|^{2} \leq g_{k+1}^{\mathrm{T}} d_{k+1} \leq-c_{1}\left\|g_{k+1}\right\|^{2},
\end{gathered}
$$

where $\delta>0, \tau>0, \rho \in(0,1)$ and $0<c_{1}<1<c_{2}$.

It is well known that some other people have studied many of the variants of the LS method, for example [3-4]. In this paper, a kind of the LS method is proposed:

$$
\beta_{k}^{\mathrm{VLS}}=-\frac{g_{k}^{\mathrm{T}}\left(g_{k}-t_{k} g_{k-1}\right)}{d_{k-1}^{\mathrm{T}} g_{k-1}},
$$

where $t_{k}=\frac{\left\|g_{k}\right\|}{\left\|g_{k-1}\right\|}$, and $\|\cdot\|$ is the Euclidean norm.

In the next section, we prove the global convergence of the new method for nonconvex functions with the Grippo-Lucidi line search. In Section 3, numerical experiments are given.

\section{Global Convergence of the New Method}

In order to prove the global convergence of the new method, we assume that the objective function satisfies 
the following assumption.

Assumption (H):

1) The level set $N=\left\{x \mid f(x) \leq f\left(x_{1}\right)\right\}$ is bounded, where $x_{1}$ is the starting point.

2) In some neighborhood $W$ of $N$, the objective function is continuously differentiable, and its gradient is Lipschitz continuous, i.e., there exists a constant $L>0$ such that

$$
\|g(x)-g(y)\| \leq L\|x-y\|, \text { for all } x, y \in W .
$$

Lemma 2.1 [5]. Suppose Assumption (H) holds. Consider any iteration in the form (1.1) and (1.2), where $d_{k}$ satisfies $g_{k}^{\mathrm{T}} d_{k}<0$ for $k \in N^{+}$and $\alpha_{k}$ satisfies Grippo-Lucidi line search. Then

$$
\sum_{k \geq 1} \cos ^{2} \theta_{k}\left\|g_{k}\right\|^{2}<+\infty .
$$

where $\cos \theta_{k}=-g_{k}^{\mathrm{T}} d_{k} /\left(\left\|g_{k}\right\| \cdot\left\|d_{k}\right\|\right)$ and $\theta_{k}$ is the angle between $-g_{k}$ and $d_{k}$.
The following Lemma shows that the Grippo-lucidi line search is suitable for the new formula.

Lemma 2.2. Suppose that Assumption (H) holds. Consider the method of form (1.1) and (1.2), where $\beta_{k}=\beta_{k}^{\mathrm{VLS}}$, and where $\alpha_{k}$ satisfies Grippo-Lucidi line search. Then $\forall k$, there exists a constant $c>0$ such that $\alpha_{k} \geq c \frac{\left|g_{k}^{\mathrm{T}} d_{k}\right|}{\left\|d_{k}\right\|^{2}}$.

Proof. Since $d_{1}=-g_{1},(1.5)$ holds for $k=1$. Suppose that (1.5) holds for $k \geq 1$.

Denote

$$
c_{3}=\frac{\min \left(1-c_{1}, c_{2}-1\right)}{2 L}>0 .
$$

By (1.2), Lipschitz condition (2.1) and (1.5), for any $\alpha_{k} \in\left(0, c_{3} \frac{\left|g_{k}^{\mathrm{T}} d_{k}\right|}{\left\|d_{k}\right\|^{2}}\right)$, we have

$$
\begin{aligned}
& \left|g_{k+1}^{\mathrm{T}} d_{k+1}+\left\|g_{k+1}\right\|^{2}\right| \leq\left|\beta_{k+1}^{V L S}\right| \cdot\left|g_{k+1}^{\mathrm{T}} d_{k}\right|=\frac{\left|g_{k+1}^{\mathrm{T}}\left(g_{k+1}-t_{k+1} g_{k}\right)\right|}{\left|-d_{k}^{\mathrm{T}} g_{k}\right|} \cdot\left|g_{k+1}^{\mathrm{T}} d_{k}\right| \leq \frac{\left\|g_{k+1}||^{2} \cdot\left(\left\|g_{k+1}-g_{k}+g_{k}-t_{k+1} g_{k}\right\|\right) \cdot\right\| d_{k} \|}{\left|-d_{k}^{\mathrm{T}} g_{k}\right|} \\
& \leq \frac{\left\|g_{k+1}\right\|^{2} \cdot\left(\left\|g_{k+1}-g_{k}\right\|+\| g_{k}-t_{k+1} g_{k}||\right) \cdot\left\|d_{k}\right\| \mid}{\left|-d_{k}^{\mathrm{T}} g_{k}\right|} \leq \frac{\left\|g_{k+1}\right\|^{2} \cdot\left(\left\|g_{k+1}-g_{k}\right\|+\mid\left\|g_{k+1}\right\|-\left\|g_{k}\right\|\right) \cdot\left\|d_{k}\right\|}{\left|-d_{k}^{\mathrm{T}} g_{k}\right|} \\
& \leq \frac{\left\|g_{k+1}\right\|^{2} \cdot 2\left\|g_{k+1}-g_{k}\right\| \cdot\left\|d_{k}\right\|}{\left|-d_{k}^{\mathrm{T}} g_{k}\right|} \leq \frac{\left\|g_{k+1}||^{2} \cdot 2 L \alpha_{k} \cdot\right\| d_{k} \|^{2}}{\left|-d_{k}^{\mathrm{T}} g_{k}\right|} \leq \min \left(1-c_{1}, c_{2}-1\right) \cdot\left\|g_{k+1}\right\|^{2} .
\end{aligned}
$$

So (1.5) holds, for any $\alpha_{k} \in\left(0, c_{3} \frac{\left|g_{k}^{\mathrm{T}} d_{k}\right|}{\left\|d_{k}\right\|^{2}}\right)$.

On the other hand, by the mean value theorem and Lipschitz condition (2.1), we have

$$
\begin{aligned}
& f\left(x_{k}+\alpha_{k} d_{k}\right)-f\left(x_{k}\right) \\
& =\int_{0}^{1} g\left(x_{k}+t \alpha_{k} d_{k}\right)^{\mathrm{T}}\left(\alpha_{k} d_{k}\right) \mathrm{d} t \\
& =\alpha_{k} g_{k}^{\mathrm{T}} d_{k}+\int_{0}^{1}\left[g\left(x_{k}+\alpha_{k} d_{k}\right)-g_{k}\right]^{\mathrm{T}}\left(\alpha_{k} d_{k}\right) \mathrm{d} t \\
& \leq \alpha_{k} g_{k}^{\mathrm{T}} d_{k}+\frac{1}{2} L \alpha_{k}^{2}\left\|d_{k}\right\|^{2} .
\end{aligned}
$$

We can test (1.4) holds, for $\alpha_{k} \in\left(0, \frac{2}{L+2 \delta} \frac{\left|g_{k}^{\mathrm{T}} d_{k}\right|}{\left\|d_{k}\right\|^{2}}\right)$.

The existence of $\alpha_{k}$ satisfying (1.4) and (1.5) has bean proved. Furthermore, the conclusion holds for $c=\min \left(\tau, c_{3}, \frac{2}{L+2 \delta}\right)$.

Theorem 2.1. Suppose that Assumption (H) holds. Consider the method of form (1.1) and (1.2), where
$\beta_{k}=\beta_{k}^{\mathrm{VLS}}$, and where $\alpha_{k}$ satisfies Grippo-Lucidi line search. Then

$$
\liminf _{k \rightarrow+\infty}\left\|g_{k}\right\|=0 \text {. }
$$

Proof. By Lipschitz condition (1.2), (1.3), (1.5) and (2.1), we can obtain

$$
\begin{aligned}
\left\|d_{k}\right\| & \leq\left\|g_{k}\right\|+\left|\beta_{k}^{\mathrm{VLS}}\right| \cdot\left\|d_{k-1}\right\| \\
& \leq\left\|g_{k}\right\| \cdot\left(1+\frac{\left\|g_{k}-t_{k} g_{k-1}\right\|}{\left|-d_{k-1}^{T} g_{k-1}\right|} \cdot\left\|d_{k-1}\right\|\right) \\
& \leq\left\|g_{k}\right\| \cdot\left(1+\frac{\left(\left\|g_{k}-g_{k-1}\right\|+\mid\left\|g_{k}\right\|-\left\|g_{k-1}\right\|\right) \cdot\left\|d_{k-1}\right\|}{\left|-d_{k-1}^{\mathrm{T}} g_{k-1}\right|}\right) \\
& \leq\left\|g_{k}\right\| \cdot\left(1+\frac{2 L \alpha_{k-1}}{\left|-d_{k-1}^{\mathrm{T}} g_{k-1}\right|}\left\|d_{k-1}\right\|^{2}\right) \\
& =(1+2 L \tau) \cdot\left\|g_{k}\right\| .
\end{aligned}
$$

By the Assumption $(\mathrm{H})$, we know that Lemma 3.1 holds. From (1.5), (2.2) and (2.4), we have 
Table 1. The performance of DY method, LS method and VLS method.

\begin{tabular}{ccccc}
\hline Problem & Dim & DY & LS & VLS \\
\hline Beale & 2 & $75 / 186 / 164$ & $18 / 65 / 55$ & $25 / 72 / 64$ \\
Box Three-Dimensional & 3 & $1 / 1 / 1$ & $1 / 1 / 1$ & $1 / 1 / 1$ \\
Penalty1 & 50 & $1727 / 2117 / 2043$ & $85 / 426 / 315$ & $65 / 112 / 98$ \\
& 100 & $31 / 157 / 121$ & $18 / 120 / 83$ & $22 / 146 / 119$ \\
& 200 & $26 / 160 / 121$ & $28 / 157 / 114$ & $20 / 124 / 93$ \\
\hline
\end{tabular}

$$
\begin{aligned}
\infty & >\sum_{k \geq 1} \cos ^{2} \theta_{k}\left\|g_{k}\right\|^{2}=\sum_{k \geq 1} \frac{\left(g_{k}^{\mathrm{T}} d_{k}\right)^{2}}{\left\|d_{k}\right\|^{2}} \\
& \geq c_{1}^{2}(1+2 L \tau)^{-2} \sum_{k \geq 1}\left\|g_{k}\right\|^{2} .
\end{aligned}
$$

This result implies $\liminf _{k \rightarrow+\infty}\left\|g_{k}\right\|=0$.

\section{Numerical Reusults}

In this section, we give the new algorithm.

Algorithm 3.1:

Step 1: Data: $x_{1} \in R^{n}, \varepsilon \geq 0$. Set $d_{1}=-g_{1}$, if $\left\|g_{1}\right\| \leq \varepsilon$, then stop.

Step 2: Compute $\alpha_{k}$ by the Grippo-Lucidi line searches.

Step 3: Let $x_{k+1}=x_{k}+\alpha_{k} d_{k}, g_{k+1}=g\left(x_{k+1}\right)$, if $\left\|g_{k+1}\right\| \leq \varepsilon$, then stop.

Step 4: Compute $\beta_{k+1}$ by (1.6), and generate $d_{k+1}$ by (1.2).

Step 5: Set $k=k+1$, go to step 2 .

We test the Algorithm 3.1 on the following problems, and compare its performance to that of the DY method and LS method with the strong Wolfe line searches where $\alpha_{k}$ is computed by

$$
\begin{gathered}
f\left(x_{k}+\alpha_{k} d_{k}\right) \leq f\left(x_{k}\right)+\delta \alpha_{k} g_{k}^{\mathrm{T}} d_{k}, \\
\left|g\left(x_{k}+\alpha_{k} d_{k}\right)^{\mathrm{T}} d_{k}\right| \leq-\sigma g_{k}^{\mathrm{T}} d_{k} .
\end{gathered}
$$

In algorithm, the parameters: $\tau=1.5, \rho=0.5$, $c_{1}=0.25, c_{2}=1.5, \delta=0.01, \sigma=0.1$. The termination condition is $\left\|g_{k}\right\| \leq 10^{-6}$, or It-max $>9999$. It-max denotes the Maximum number of iterations.

The numerical results of our tests are reported in Table 1. The column "Problem" represents the problem's name; "Dim" denotes the dimension of the tested problems. The detailed numerical results are listed in the form NI/NF/NG, where NI, NF, NG denote the number of iterations, function evaluations, and gradient evaluations, respectively.

VLS method: $\beta_{k}=\beta_{k}^{\mathrm{VLS}}, \alpha_{k}$ by the Grippo-Lucidi line searches
LS method: $\beta_{k}=\beta_{k}^{\mathrm{LS}}, \alpha_{k}$ by the strong Wolfe line searches.

DY method: $\alpha_{k}$ by the strong Wolfe line searches, $\beta_{k}$ is computed by $\beta_{k}^{\mathrm{LS}}=\frac{\left\|g_{k}\right\|^{2}}{d_{k-1}^{\mathrm{T}}\left(g_{k}-g_{k-1}\right)}$.

In the following, we give the tested functions:

1) Beale Test Function:

$$
\begin{aligned}
f(x)= & {\left[1.5-x_{1}\left(1-x_{2}\right)\right]^{2}+\left[2.25-x_{1}\left(1-x_{2}^{2}\right)\right]^{2} } \\
& +\left[2.625-x_{1}\left(1-x_{2}^{3}\right)\right]^{2},
\end{aligned}
$$

the initial point $(1,1)^{\mathrm{T}}$.

2) Box Three-Dimensional Test Function:

$$
f(x)=\sum_{i=1}^{3}\left[\mathrm{e}^{-0.11 x_{1}}-\mathrm{e}^{-0.11 x_{2}}-x_{3}\left(\mathrm{e}^{-0.1 i}-\mathrm{e}^{-i}\right)\right]^{2},
$$

the initial point $(0,10,20)^{\mathrm{T}}$.

3) Penalty Test Function I:

$$
f(x)=10^{-5} \sum_{i=1}^{n}\left(x_{i}-1\right)^{2}+\left(\sum_{i=1}^{n} x_{i}^{2}-0.25\right)^{2},
$$

the initial point $(1,2, \cdots, m)^{\mathrm{T}}$.

From the numerical results, we know that the new method is efficient for the given problems under the Grippo-Lucidi line searches.

\section{Acknowledgements}

We are grateful to anonymous referees and editors for their useful suggestions and comments on this paper.

\section{References}

[1] Y. Liu and C. Storey, "Efficient Generalized Conjugate Gradient Algorithms. Part 1: Theory," Journal of Optimization Theory and Applications, Vol. 69, No. 1, 1992, pp. 129-137. doi:10.1007/BF00940464

[2] Z. F. Li, J. Chen and N. Y. Deng, "A New Conjugate Gradient Method and Its Global Convergence Properties," Mathematical Programming, Vol. 78, 1997, pp. 375-391. doi:10.1007/BF02614362 
[3] G. H. Yu, Y. L. Zhao and Z. X. Wei, "A Descent Nonlinear Conjugate Gradient Method for Large-Scale Unconstrained Optimization," Applied Mathematics and Computation, Vol. 187, No. 2, 2007, pp. 636-643.

doi:10.1016/j.amc.2006.08.087

[4] J. K. Liu, X. L. Du and K. R. Wang, "Convergence of Descent Methods with Variable Parameters," Acta
Mathematicae Applicatae Sinica, Vol. 33, No. 2, 2010, pp. 222-232.

[5] Z. F. Li, J. Chen and N. Y. Deng, "Convergence Properties of Conjugate Gradient Methods with Goldstein Line Searches," Journal of China Agricultural University, Vol. 1, No. 4, 1996, pp. 15-18. 